\title{
The impact of generalized joint laxity (GJL) on the posterior neck pain, cervical disc herniation, and cervical disc degeneration in the cervical spine
}

\author{
Sun-Mi Lee, MD ${ }^{\mathrm{a}}$, Su Chan Oh, MD ${ }^{\mathrm{b}}$, Jin S. Yeom, MD ${ }^{\mathrm{b}}$, Ji-Hoon Shin, $\mathrm{MD}^{\mathrm{c}}$, \\ Sam-Guk Park, $\mathrm{MD}^{\mathrm{c}}$, Duk-Seop Shin, $\mathrm{MD}^{\mathrm{c}}$, Myun-Whan Ahn, $\mathrm{MD}^{\mathrm{c}}$, Gun Woo Lee, MD ${ }^{\mathrm{c} *}$ \\ ${ }^{a}$ Department of Family Medicine, Dongkang Hospital, 239, Taehwa-ro, Jung-gu, Ulsan 44455, Korea \\ ${ }^{\mathrm{b}}$ Spine Center and Department of Orthopaedic Surgery, Seoul National University College of Medicine and Seoul National University Bundang Hospital, 82, \\ Gumi-ro 173beon-gil, Bundang-gu, Seongnam-si, Gyeonggi-do 13620, Korea \\ ${ }^{\mathrm{c}}$ Department of Orthopaedic Surgery, Yeungnam University Hospital, 170, Hyeonchung-ro, Nam-gu, Daegu 42415, Korea \\ Received 13 November 2015; revised 23 June 2016; accepted 2 August 2016
}

Abstract

BACKGROUND CONTEXT: Generalized joint laxity (GJL) can have a negative impact on lumbar spine pathology, including low back pain, disc degeneration, and disc herniation, but the relationship between GJL and cervical spine conditions remains unknown.

PURPOSE: To investigate the relationship between GJL and cervical spine conditions, including the prevalence of posterior neck pain (PNP), cervical disc herniation $(\mathrm{CDH})$, and cervical disc degeneration (CDD), in a young, active population.

STUDY DESIGN: Retrospective 1:2 matched cohort (case-control) study from prospectively collected data

PATIENT SAMPLE: Of a total of 1853 individuals reviewed, 73 individuals with GJL (study group, gruop A) and 146 without GJL (control group, Group B) were included in the study according to a 1:2 case-control matched design for age, sex, and body mass index.

OUTCOME MEASURE: The primary outcome measure was the prevalence and intensity of PNP at enrollment based on a visual analogue scale score for pain. The secondary outcome measures were (1) clinical outcomes as measured with the neck disability index (NDI) and 12-item short form health survey (SF-12) at enrollment, and (2) radiological outcomes of CDH and CDD at enrollment.

METHODS: We compared baseline data between groups. Descriptive statistical analyses were performed to compare the 2 groups in terms of the outcome measures.

RESULTS: The prevalence and intensity of PNP were significantly greater in group A (patients with GJL) than in group B (patients without GJL) (prevalence: $\mathrm{p}=.02$; intensity: $\mathrm{p}=.001$ ). Clinical outcomes as measured with NDI and SF-12 did not differ significantly between groups. For radiologic outcomes, the prevalence of CDD was significantly greater in group A than in group $B(p=.04)$, whereas the prevalence of $\mathrm{CDH}$ did not differ significantly between groups $(\mathrm{p}=.91)$.

CONCLUSIONS: The current study revealed that GJL was closely related to the prevalence and intensity of PNP, suggesting that GJL may be a causative factor for PNP. In addition, GJL may contribute to the occurrence of CDD, but not CDH. Spine surgeons should screen for GJL in patients

FDA device/drug status: Not applicable.

Author disclosures: $\boldsymbol{S}-\mathbf{M L}$ : Nothing to disclose. SCO: Nothing to disclose. JSY: Speaking and/or Teaching Arrangements: Medtronic (B), outside the submitted work; Grant: CG Bio (B, Paid to the institution), outside the submitted work. $\boldsymbol{J}$-HS: Nothing to disclose. $\boldsymbol{S}$-GP: Nothing to disclose. $\boldsymbol{D}-\boldsymbol{S S}$ : Nothing to disclose. $\boldsymbol{M}$-WA: Nothing to disclose. $\boldsymbol{G W L}$ : Nothing to disclose.

The disclosure key can be found on the Table of Contents and at www.TheSpineJournalOnline.com.
Funding: No funding was received in support of this work. No benefits in any form have been or will be received from a commercial party related directly or indirectly to the subject of this manuscript.

* Corresponding author. Department of Orthopaedic Surgery, Yeungnam University Hospital, 170, Hyeonchung-ro, Nam-gu, Daegu, 42415, Republic of Korea. Tel.: +8253620 3640; fax: +8253628 4020 .

E-mail address: gwlee1871@gmail.com (G.W. Lee). 
with PNP and inform patients of its potential negative impact on disc degeneration of the cervical spine. (c) 2016 Elsevier Inc. All rights reserved.

Keywords: $\quad$ Cervical spine; Clinical outcomes; Disc degeneration; Disc herniation; General joint laxity; Posterior neck pain

\section{Introduction}

Recent studies have revealed that general joint laxity (GJL) is associated with musculoskeletal disorders and related clinical manifestations [1-6], underscoring the need to regard GJL as a disease with pathologic sequelae, rather than just a benign condition.

The relationship between GJL and musculoskeletal disorders is well documented, and includes shoulder instability, ligamentous injury of the knee joint, and traumatic lesions of the ankle and elbow [2,3,7-13]. Some studies have demonstrated that certain lumbar spine pathologies are also associated with GJL, including aggravated lower back pain, segmental hypermobility at the lumbar spine, accelerated lumbar disc degeneration, and lumbar disc herniation [6,14-18]. GJL is also negatively associated with outcomes of injection treatment for lumbar disc herniation $[16,18]$. Although the negative impact of GJL on pathologic conditions affecting the lumbar spine has been well documented, its influence on problems of the cervical spine has not yet been thoroughly described.

The present study investigated the relationship between GJL and cervical spine pathologies. We evaluated the relationship between GJL and the prevalence of posterior neck pain (PNP), cervical disc herniation ( $\mathrm{CDH})$, and cervical disc degeneration (CDD), using a 1:2 matched cohort design in a young, active population with and without GJL.

\section{Methods}

\section{Participants}

The present study was approved by the corresponding author's institutional review board. We aimed to determine the significance and features of GJL in a young population between 20 and 30 years old, and we prospectively collected data after informed consent of enrolled patients in an outpatient orthopedic clinic from January 2012. In the current study, we investigated the impact of GJL on the prevalence of PNP, CDH, and CDD in a young, active population, for which we conducted the present study using a retrospective 1:2 matched cohort (case-control) study of the prospectively collected data.

The present study included individuals between 20 and 30 years of age who provided informed consent to participate. Patients were excluded if they had a history of conservative or surgical treatment at the cervical spine, had a history of tumor or infection, or were unable to accurately respond to pre- and postoperative questionnaires due to a history of stroke, dementia, or major medical illness that required intensive treatment. All patients were fully informed before enrollment about the study's purpose and research course. Between January
2012 and December 2014, our research coordinators distributed 4 questionnaires to these patients at our outpatient clinic, and cervical spine radiographs (anteroposterior and lateral, as well as flexion and extension dynamic images) and magnetic resonance imaging (MRI) were performed after informed consent and approval.

\section{Patients}

A total of 1,853 individuals met the inclusion and exclusion criteria for the present study, of whom 73 individuals had GJL based on the diagnostic criteria described below. Thus, the study group (Group A) included 73 individuals with GJL, and the control group (Group B) included 146 individuals without GJL, based on a 1:2 case-control matched design for age, sex, and body mass index to control for demographic bias.

\section{Definition of GJL}

The Beighton scale was used to determine whether GJL was present, and an assessment of the joint range of motion was performed with a standard clinical set of goniometers (Table 1). A threshold cutoff value of 4 out of 9 points was used to define the presence of GJL, based on the results of previous studies [17-19].

\section{Outcome measures}

The primary outcome measures were the prevalence and intensity of PNP based on a visual analogue scale (VAS) score for pain at the time of enrollment. Patients were instructed to make a mark on a horizontally oriented, 10-point VAS score sheet, with "no pain" on the far left of the sheet and "greatest pain possible" on the far right of the sheet. The records were collected and analyzed by a research coordinator who was otherwise not involved in the study.

The secondary outcome measures were (1) clinical outcomes and (2) radiologic outcomes. Clinical outcomes were evaluated with the neck disability index and a 12-item short form health survey (SF-12) at the time of enrollment. The SF-12 questionnaire consists of the physical component summary (PCS) and mental component summary (MCS); scores for the PCS and MCS were recorded and evaluated separately. Radiologic evaluation was performed with plain radiographs and MRI obtained at the time of enrollment, with a focus on disc herniation and degeneration in the cervical spine. Disc herniation was classified according to 4 characteristics: level, side (left or right), location (central, paracentral, foraminal), and type (bulging, protrusion, extrusion, sequestration). Disc degeneration was evaluated at 6 cervical 


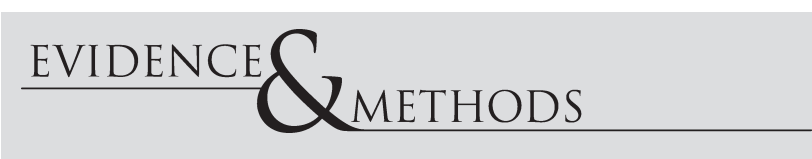

\section{Context}

The association between generalized joint laxity (GJL) and lumbar spine issues is well known. However, its association with cervical spinal disorders has not been explored to a great extent. In this context, the authors present a retrospective matched cohort study developed from data collected at their center.

\section{Contribution}

This study matched 73 patients with GJL to 146 without the condition. The authors maintain that GJL was associated with increased rates of posterior neck pain and cervical degeneration but not disc herniation.

\section{Implications}

The results of this study yield some interesting associations but they are unable to speak to causation given design and study limitations, including clear selection bias. This study was only able to evaluate patients who were identified with GJL and who also happened to present to a spine clinic for evaluation and treatment. This cannot be considered commensurate to a natural history study of patients with GJL. However the data may have been collected, this remains a retrospective study with most of the inherent limitations associated with such work. The level of evidence for this effort should be seen as grade IV as a result. - The Editors

Table 1

Beighton scale

\begin{tabular}{llll}
\hline Criteria & Yes & No \\
\hline $\begin{array}{l}\text { 1. } \\
\quad \begin{array}{l}\text { Passive dorsiflexion of fifth finger beyond } 90^{\circ} \text { at } \\
\text { metacarpophalangeal joint }\end{array}\end{array}$ & $1^{*}$ & 0 \\
2. Passive apposition of thumb to flexor aspect of forearm & $1^{*}$ & 0 \\
3. Hyperextension of elbow beyond $10^{\circ}$ & $1^{*}$ & 0 \\
4. Hyperextension of knee beyond $10^{\circ}$ & $1^{*}$ & 0 \\
5. Forward flexion of the trunk with the knees fully extended & 1 & 0 \\
$\quad$ so that the palms of the hands rest flat on the floor & 9 & \\
Total $\quad$ & 9
\end{tabular}

A threshold cutoff value of 4 in total 9 points was used to classify generalized joint laxity as present.

* 1 point each side. intervertebral discs (C2/3, C3/4, C4/5, C5/6, C6/7, and C7/ T1) for all enrolled participants, and the grade of disc degeneration (Grades I-V) was classified with the MRI-based grading system of Miyazaki et al. [20], which evaluates 4 factors: nucleus signal intensity, nucleus structure, the distinction between the nucleus pulposus and annulus fibrosus, and disc height (Table 2). The mean \pm SD of the six disc grades was calculated for each participant, and the values were compared between the 2 groups. Clinical records were analyzed by a research coordinator, and radiologic outcomes were analyzed by a surgeon who was not otherwise involved in the study.

\section{Statistical analysis}

Descriptive statistical analyses were performed to compare the 2 groups (with or without GJL) via the Student $t$ test for continuous data and Fisher's exact test for categorical data. Analysis of variance was also used to compare the means between groups. Data are presented as mean $\pm \mathrm{SD}$ when applicable. SAS version 9.1.3 (SAS Institute Inc., Cary, NC, USA) was used for all analyses, and two-tailed p-values $<.05$ were considered statistically significant.

\section{Results}

\section{Demographic data}

We retrospectively reviewed 73 individuals with GJL (Group A) and 146 individuals without GJL (Group B, control) who met the inclusion and exclusion criteria. We used a 1:2 matched design. Demographic characteristics, including age, sex, smoking history, and previous illnesses such as diabetes mellitus, hypertension, and thyroid disease, did not differ significantly between the 2 groups (Table 3 ).

\section{Prevalence and severity of PNP}

The prevalence of PNP was $30.1 \%$ (22/73; $95 \%$ CI 20 40 ) in group A (with GJL) and $15.7 \%$ (23/146; 95\% CI 9.821.6) in group $B$ (without GJL), with a significant difference between the 2 groups $(\mathrm{p}=.02)$. The intensity of PNP was $38.6 \pm 8.3$ in group A and 29.6 \pm 11.4 in group $B$, which was also significantly different between groups $(\mathrm{p}=.001)$. The percentage of participants with PNP who had a VAS score greater than 40 and who required pain medication was higher in group A $(11 / 73,15.1 \%$; $95 \%$ CI $6.9-23.3)$ than in group B $(9 / 146$, $6.2 \%$; 95\% CI 2.3-10.1) ( $\mathrm{p}=.04)$.

Table 2

Grading system for disc degeneration of the cervical spine

\begin{tabular}{lllll}
\hline Grade & Nucleus signal intensity & Nucleus structure & Distinction of nucleus and annulus & Disc height \\
I & Hyperintense & Homogenous white & Clear & Normal \\
II & Hyperintense & Inhomogenous with horizontal band, white & Clear & Normal \\
III & Intermediate & Inhomogenous, gray to black & Unclear & Normal to decrease \\
IV & Hypointense & Inhomogenous, gray to black & Lost & Normal to decrease \\
V & Hypointense & Inhomogenous, gray to black & Lost & Collapsed \\
\hline
\end{tabular}


Table 3

Demographic data of enrolled patients

\begin{tabular}{lccr}
\hline & $\begin{array}{l}\text { Group A } \\
\text { (study group) }\end{array}$ & $\begin{array}{l}\text { Group B } \\
\text { (control group) }\end{array}$ & $P$ \\
\hline $\mathrm{N}$ & 73 & 146 & - \\
Age (year) & $26.3 \pm 4.8$ & $25.9 \pm 5.3$ & .87 \\
Sex (male/female) & $52 / 21$ & $101 / 45$ & .88 \\
Height $(\mathrm{cm})$ & $169.4 \pm 15.2$ & $168.2 \pm 13.1$ & .74 \\
Weight $(\mathrm{kg})$ & $67.3 \pm 9.1$ & $66.5 \pm 8.3$ & .81 \\
BMI $\left(\mathrm{kg} / \mathrm{m}^{2}\right)$ & $25.8 \pm 5.2$ & $26.4 \pm 6.9$ & .77 \\
Smoking $(\%)$ & $12(16.4)$ & $23(15.8)$ & 1.00 \\
Previous illness & & & - \\
$\quad$ DM & 1 & 1 & \\
$\quad$ HTN & 0 & 2 & \\
Thyroid disease & 1 & 0 & \\
\hline
\end{tabular}

BMI, body mass index; DM, diabetes mellitus; GJL, generalized joint laxity; HTN, hypertension.

Group A, individuals with GJL; Group B, individuals without GJL.

Values in data cells represent mean $\pm \mathrm{SD}$.

\section{Secondary outcome measures}

The neck disability index was $16.3 \pm 7.5$ in group A and $14.7 \pm 9.2$ in group $\mathrm{B}$, with no significant difference between groups $(\mathrm{p}=.26)$. For the SF-12, the mean PCS score was $43.1 \pm 12.7$ in group $A$ and $42.7 \pm 14.1$ in group $B(p=.58)$, and the mean MCS score was $49.7 \pm 11.5$ in group $A$ and $50.6 \pm 11.8$ in group $\mathrm{B}(\mathrm{p}=.71)$.

Detailed outcomes on disc herniation and disc degeneration are summarized in Tables 4 and 5. The prevalence of disc herniation was $7.1 \%$ (31/438) in group A and 6.8\% (60/ 876 ) in group $B$, with no significant difference between groups $(p=.91)$. The most common herniated type and segment was a paracentrally located bulging disc at $\mathrm{C} 4 / 5$. The mean grade of disc degeneration did not differ significantly between groups

Table 4

Detailed information of disc herniation in the cervical spine

\begin{tabular}{lccc}
\hline & $\begin{array}{l}\text { Group A } \\
(\mathrm{n}=73)\end{array}$ & $\begin{array}{l}\text { Group B } \\
(\mathrm{n}=146)\end{array}$ & $P$ \\
\hline Level & & & \\
C2/3 & 0 & 0 & \\
C3/4 & 1 & 3 \\
C4/5 & 13 & 19 \\
C5/6 & 10 & 13 & \\
C6/7 & 7 & 3 & - \\
C7/T1 & 0 & 37 \\
Side & & 23 \\
Left & 21 & \\
Right & 10 & 9 \\
Location & & 38 \\
Central & 3 & 13 \\
Paracentral & 21 & \\
Foraminal & 7 & 37 \\
Type & & 19 \\
Bulging & 19 & 4 \\
Protrusion & 10 & 0 \\
Extrusion & 2 & \\
Sequestration & 0 & \\
\hline
\end{tabular}

GJL, generalized joint laxity.

Group A, individuals with GJL; Group B, individuals without GJL.
Table 5

Detailed information of disc degeneration in the cervical spine

\begin{tabular}{lccc}
\hline & $\begin{array}{l}\text { Group A } \\
(\mathrm{n}=73)\end{array}$ & $\begin{array}{l}\text { Group B } \\
(\mathrm{n}=146)\end{array}$ & $P$ \\
\hline Level & & & \\
$\mathrm{C} 2 / 3$ & 0 & 0 & \\
$\mathrm{C} 3 / 4$ & 2 & 3 & \\
$\mathrm{C} 4 / 5$ & 10 & 13 & - \\
$\mathrm{C} 5 / 6$ & 16 & 18 & - \\
$\mathrm{C} 6 / 7$ & 9 & 12 & $.04^{*}$ \\
$\mathrm{C} 7 / \mathrm{T} 1$ & 1 & 3 & .87 \\
Total & 38 & 49 & \\
Mean value & $1.4 \pm 1.1$ & $1.4 \pm 0.8$ & \\
\hline \multicolumn{4}{c}{ GJL, generalized joint laxity. } \\
Group A, individuals with GJL; Group B, individuals without GJL. \\
* p $<$.05.
\end{tabular}

(group A: $1.4 \pm 1.1$; group B: $1.4 \pm 0.8 ; \mathrm{p}=.87$ ), but the prevalence of disc degeneration was higher in group A $(8.7 \%, 38$ / $438 ; 95 \%$ CI, 6.1-11.3) than in group B $(5.6 \%, 49 / 876 ; 95 \%$ CI 4.1-7.1) in group B ( $\mathrm{p}=.04)$. Degeneration was most common in segment $\mathrm{C} 5 / 6$, followed by $\mathrm{C} 4 / 5, \mathrm{C} 6 / 7$, and $\mathrm{C} 3 / 4$.

\section{Discussion}

Once considered a benign condition, GJL has recently been associated with pathologic conditions, especially musculoskeletal problems including ligament and muscle injury around the knee and shoulder joint $[1-13,21]$. Furthermore, spine clinicians have documented a relationship between GJL and lumbar spine pathologies, including a higher incidence of low back pain, hypermobility at the lumbar segments, accelerated disc degeneration and disc herniation, aggravated adjacent segment problems after lumbar fusion surgery, and worse treatment outcomes [6,13-18,22]. However, the impact of GJL on cervical spine pathology has not been thoroughly described. Thus, we investigated the relationship between GJL and cervical spine pathologies, focusing on PNP, CDH, and CDD in a young, active population.

The present study is the first to thoroughly evaluate the impact of GJL on conditions affecting the cervical spine. The prevalence and severity of PNP were significantly greater in individuals with GJL than in those without GJL, similar to the results of lumbar spine studies [14-18]. The percentage of individuals with PNP who had a score of 40 or more on the VAS score and who required pain medication was significantly greater among individuals with GJL than those without GJL. As such, GJL of the cervical spine may be a predictive factor for PNP. Another major finding of the present study was that radiologic evaluation revealed that disc herniation in the cervical spine was not related to the presence of GJL. The type and location of disc herniation did not differ significantly between patients with and without GJL. However, disc degeneration in the cervical spine might be associated with GJL. The prevalence of disc degeneration was significantly greater in patients with GJL than in those without GJL, although the mean grade of disc degeneration did not differ. These results suggest that baseline GJL 
may be a negative prognostic factor for cervical disc degeneration. The mixed results for disc degeneration (prevalence vs. mean grade value) may be explained by 2 factors related to the study design. First, the statistical analysis was performed on the number of cervical segments, not the number of enrolled individuals, which decreased the percentage of degenerative lesions and led to a convergence in numerical values between the 2 groups. Second, we conducted the present study in a young population with a low prevalence of degenerative lesions of the cervical spine, despite the presence of GJL. Therefore, we believe that the outcomes related to prevalence have more clinical relevance than the mean values for degeneration. On the basis of the outcomes of the present study, we suggest that GJL with PNP should be regarded as a pathologic condition that requires proper management. In addition, spine surgeons should screen for GJL in patients with PNP and inform them of its potential negative contribution to disc degeneration of the cervical spine.

The present study has several limitations. First, this was a retrospective case-control study with a relatively small sample size. To better establish the impact of GJL on the cervical spine, more patients need to be studied with prospectively collected data. Second, the participants in our study were young individuals between the ages of 20 and 30 . Because the prevalence of GJL decreases with age from childhood, we limited the present study to young individuals to minimize the confounding effects of age. However, this limitation could have made the statistical analysis more difficult to interpret, given the abnormal distribution of patients. Further studies should be performed with patients of all ages. Third, we did not study the differentiation of range of motion at the cervical spine in dynamic extension or flexion lateral radiographs, which could have informed our understanding of GJL. Further study with cervical range of motion in dynamic radiographs is necessary to better understand GJL. Fourth, 95\% CI values for the 3 prevalence percentages (PNP prevalence, prevalence more than 40 VAS score, and disc degeneration prevalence) were overlapped, which may lead to be misunderstood as lowering or not having the statistical significance. However, the overlapping can be caused by some reasons such as small sample size and selection bias from population, and because significant differences with p-value of less than .05 between 2 groups were proven by the statistical analysis, the overlapping can be ignored in interpreting the results. To better determine it, further studies with larger sample size should be necessary. Finally, we used the Beighton scale with the diagnostic criterion of 4 points or more to diagnose GJL. The gold standard for the diagnosis of GJL has not been established, but previous studies have demonstrated that the Beighton scale with a cutoff of 4 points has optimal accuracy with great sensitivity, specificity, and reliability in comparison with other methods and criteria [4,5,19,23,24].

Despite these limitations, we believe that the present study has unique strengths. First, this is the first study to investigate the impact of GJL on cervical spine problems, including PNP, CDH, and CDD. In addition, the present study demonstrated for the first time that GJL could be a predisposing factor for the prevalence and severity of PNP and may be related to accelerated CDD. Additional studies should be performed with larger sample sizes to better establish the relationship between GJL and cervical spine pathologies.

\section{Conclusions}

To our knowledge, this is the first study to investigate the relationship between GJL and pathologies of the cervical spine, such as PNP, CDD, and CDH. The current study revealed that GJL was closely related to the prevalence and intensity of PNP, suggesting that it may be a causative factor for PNP. In addition, GJL may be a potential risk factor for CDD, but not CDH. Spine surgeons should screen for GJL in patients with PNP and inform them of its potential negative impact on disc degeneration of the cervical spine. Additional studies should be performed with a larger sample size and prospectively collected data to better establish the relationship between GJL and cervical spine pathology.

\section{Acknowledgment}

We thank and acknowledge Dr. Sung-Jun Kim, a physician assistant, for contributions to the project.

\section{References}

[1] Simpson MR. Benign joint hypermobility syndrome: evaluation, diagnosis, and management. J Am Osteopath Assoc 2006;106:531-6.

[2] Jensen BR, Olesen AT, Pedersen MT, Kristensen JH, Remvig L, Simonsen EB, et al. Effect of generalized joint hypermobility on knee function and muscle activation in children and adults: knee motor control and joint hypermobility. Muscle Nerve 2013;48:762-9.

[3] Bin Abd Razak HR, Bin Ali N, Howe TS. Generalized ligamentous laxity may be a predisposing factor for musculoskeletal injuries. J Sci Med Sport 2014;17:474-8.

[4] Simmonds JV, Keer RJ. Hypermobility and the hypermobility syndrome. Man Ther 2007;12:298-309.

[5] Hakim A, Grahame R. Joint hypermobility. Best Pract Res Clin Rheumatol 2003;17:989-1004.

[6] Castori M, Morlino S, Grammatico P. Towards a re-thinking of the clinical significance of generalized joint hypermobility, joint hypermobility syndrome, and Ehlers-Danlos syndrome, hypermobility type. Am J Med Genet A 2014;164:588-90.

[7] Ranalletta M, Bongiovanni S, Suarez F, Ovenza JML, Maignon G. Do patients with traumatic recurrent anterior shoulder instability have generalized joint laxity? Clin Orthop Relat Res 2012;470:957-60.

[8] Smith TO, Jerman E, Easton V, Bacon H, Armon K, Poland F, et al. Do people with benign joint hypermobility syndrome (BJHS) have reduced joint proprioception? A systematic review and meta-analysis. Rheumatol Int 2013;33:2709-16.

[9] Pacey V, Nicholson LL, Adams RD, Munn J, Munns CF. Generalized joint hypermobility and risk of lower limb joint injury during sport: a systematic review with meta-analysis. Am J Sports Med 2010;38:148797.

[10] Scheper MC, de Vries JE, de Vos R, Verbunt J, Nollet F, Engelbert $\mathrm{RH}$. Generalized joint hypermobility in professional dancers: a sign of talent or vulnerability? Rheumatology 2012;52:651-8.

[11] Chahal J, Leiter J, McKee MD, Whelan DB. Generalized ligamentous laxity as a predisposing factor for primary traumatic anterior shoulder dislocation. J Shoulder Elbow Surg 2010;19:1238-42.

[12] Vaishya R, Hasija R. Joint hypermobility and anterior cruciate ligament injury. J Orthop Surg 2013;21:182-4. 
[13] Bridges AJ, Smith E, Reid J. Joint hypermobility in adults referred to rheumatology clinics. Ann Rheum Dis 1992;51:793-6.

[14] Kim HJ, Yeom JS, Lee DB, Kang KT, Chang BS, Lee CK. Association of benign joint hypermobility with spinal segmental motion and its clinical implication in active young males. Spine 2013;38:E1013-19.

[15] Kim KT, Park SW, Kim YB. Disc height and segmental motion as risk factors for recurrent lumbar disc herniation. Spine 2009;34: 2674-8.

[16] Han WJ, Kim HB, Lee GW, Choi JH, Jo WJ, Lee SM. Generalized joint laxity is associated with primary occurrence and treatment outcome of lumbar disc herniation. Korean J Fam Med 2015;36:141-5.

[17] Lee SM, Lee GW. The impact of generalized joint laxity on the clinical and radiological outcomes of single-level posterior lumbar interbody fusion. Spine J 2015;15:809-16.

[18] Lee GW, Lee SM, Suh BG. The impact of generalized joint laxity on the occurrence and disease course of primary lumbar disc herniation. Spine J 2015;15:65-70

[19] Remvig L, Jensen DV, Ward RC. Are diagnostic criteria for general joint hypermobility and benign joint hypermobility syndrome based on reproducible and valid tests? A review of the literature. J Rheumatol 2007;34:798-803.
[20] Miyazaki M, Hong SW, Yoon SH, Morishita Y, Wang JC. Reliability of a magnetic resonance imaging-based grading system for cervical intervertebral disc degeneration. J Spinal Disord Tech 2008;21:288-92.

[21] Nikolajsen H, Larsen PK, Simonsen EB, Alkjær T, Falkerslev S, Kristensen JH, et al. Gait pattern in 9-11-year-old children with generalized joint hypermobility compared with controls; a crosssectional study. BMC Musculoskelet Disord 2013;14:341.

[22] Axelsson P, Karlsson BS. Intervertebral mobility in the progressive degenerative process. A radiostereometric analysis. Eur Spine J 2004; 13:567-72.

[23] Juul-Kristensen B, Rogind H, Jensen DV, Remvig L. Inter-examiner reproducibility of tests and criteria for generalized joint hypermobility and benign joint hypermobility syndrome. Rheumatology 2007;46:1835-41.

[24] Remvig L, Flycht L, Christensen KB, Juul-Kristensen B. Lack of consensus on tests and criteria for generalized joint hypermobility, Ehlers-Danlos syndrome: hypermobile type and joint hypermobility syndrome. Am J Med Genet A 2014;164:591-6. 